\title{
COVID-19, recomendaciones para el manejo farmacológico
}

\author{
COVID-19, recommendations for pharmacological management
}

COVID-19, es la peor pandemia del siglo XXI que hayamos vivido. Al 28 de febrero del año 2021, el SARS-CoV-2 ha infectado a 114 millones de personas y 2,5 millones de ellas han fallecidas en todo el mundo. Chile, no ha estado exento de esta crisis sanitaria, social y económica, alcanzando a tener 824.625 habitantes infectados y 20.572 fallecidos por esta enfermedad ${ }^{\prime}$. COVID-19 fue la primera causa de muerte en nuestro país el año $2020^{2}$. El nuevo coronavirus ha golpeado con especial fuerza a los segmentos más vulnerables de la población: los adultos mayores ${ }^{3}$ y los más pobres ${ }^{4}$.

En marzo del año 2020 y frente a las malas noticias de que la pandemia se extendía por Europa dejando miles de muertos y la detección de los primeros casos en América Latina, dio pie para comenzar a utilizar una variedad de medicamentos, con la esperanza que fueran útiles contra el SARS-CoV-2. No obstante, su uso se basó en datos de laboratorio, prepublicaciones, o estudios observacionales. Un ejemplo de ello es la hidroxicloroquina (HCQ), un medicamento antimalárico que también es utilizado como antiinflamatorio en patologías reumatológicas, como el lupus eritematoso sistémico y la artritis reumatoide. Este medicamento comenzó su uso propiciado por una carta publicada en la revista BioSciencie Trend ${ }^{5}$, donde comentan someramente, sobre una conferencia de expertos en China (realizada el 15 de febrero, 2020), país en el que se comunican los resultados favorables de HCQ en 100 pacientes con COVID-19 comparados con un grupo control. Poco después, Gautret y cols. ${ }^{6}$ publicaron un estudio pequeño $(n=36)$, no randomizado, abierto, de pacientes hospitalizados no graves COVID-19. Ellos encontraron una negativizacion de la PCR para COVID-19, de muestra nasofaríngea al 5to día, en todos los pacientes que recibieron de tratamiento HCQ y azitromicina comparado con solo HCQ o placebo. Este artículo, el más referenciado de la época, actualmente no se encuentra disponible en la web y fue modificado. Por otro lado, el entonces presidente de los Estados Unidos, Donald Trump publicitó, con cobertura periodistica nacional e internacional, las supuestas bondades que tiene la HCQ. Tal es así, que la FDA aprobó de emergencia el uso de HCQ y cloroquina para COVID-19, con el argumento de que "aunque actualmente no hay tratamientos aprobados para COVID-19, ambos fármacos han mostrado actividad en estudios de laboratorio contra coronavirus, incluido el SARSCoV-2". También, surgieron guias de una prestigiosa revista de enfermedades respiratorias con opinión de expertos, donde se recomendaba el uso de este medicamento en pacientes con COVID-19 graves, pero con baja fuerza de la evidencia ${ }^{8}$.

Este ejemplo y el caso de otros fármacos, dan cuenta de la desesperada y frenética carrera por contar con un medicamento eficaz contra el SARS-CoV-2. Pero a su vez, evidencia la falta de prolijidady de un debido proceso editorial por pares de algunas revistas cientificas al momento de publicar sobre COVID-19. Argumentando, la necesidad de contar de manera inmediata con evidencias y resultados fiables. Tal es así que más de una veintena de artículos sobre la COVID-19 han sido retractados, según Retraction Watch ${ }^{9}$. Algunos de los estudios retirados habian sido publicados en revistas muy prestigiosas como: Lancet y New England Journal of Medicine. La existencia de un posible sesgo editorial al publicar artículos sobre COVID-19 en revistas de alto impacto, ha sido también comentada en una editorial previa de esta revista sobre investigación biomédica en tiempos de pandemia ${ }^{10}$.

En ese contexto, la Sociedad Chilena de Enfermedades Respiratorias representada por nuestra presidenta la Dra. Laura Mendoza, recibió una invitación de la Dra. Daniela Pavez, coordinadora del 'Comité Antimicrobianos' de la Sociedad Chilena de Infectología para integrar junto a otros miembros de la Sociedad Chilena de Medicina Intensiva, un grupo de trabajo para desarrollar guías sobre el manejo de COVID-19. El Dr. Raúl Riquelme Oyarzún y el suscrito, aceptamos este importante desafio 
y comenzamos un trabajo periódico con reuniones online, donde analizábamos cuidadosamente la evidencia cientifica publicada respecto de distintos fármacos y el balance riesgo-beneficio durante su utilización. Después de varios meses de mancomunado trabajo de las tres ya mencionadas sociedades cientificas nacionales-SOCHINEF, SOCHIMI y SERChile- se completó el documento que lleva por titulo "Recomendaciones para el estudio y manejo farmacológico en pacientes adultos con sospecha de infección por SARS-CoV-2 (COVID-19)" "II. Estamos ciertos que la evidencia actual es limitada y sujeta a cambios y actualizaciones, por lo que las recomendaciones pueden variar y de hecho se han modificado, por cuanto esta recomendación corresponde a la $3^{\text {ra }}$ versión. Estas guías, que se publican en este número de la Revista Chilena de Enfermedades Respiratorias ${ }^{11}$, también serán publicadas en las revistas respectivas de las otras dos sociedades.

Este es un documento muy completo, que comienza con una introducción, luego continúa con un análisis de los métodos diagnósticos de infección por SARS-CoV-2 y los criterios de hospitalización. Prosigue con el estudio inicial de pacientes hospitalizados con COVID-19 y el manejo inicial general. El tratamiento farmacológico se divide en no especifico que incluye la terapia con corticoides y anticoagulante y el tratamiento farmacológico especifico, donde se evalúan los medicamentos con actividad antiviral, la inmunoterapia y los anticuerpos neutralizantes.

El análisis farmacológico específico va acompañado de tablas que contienen un resumen global de la información de los estudios clínicos aleatorizados, si están disponibles y finalizan con una recomendación precisa del respectivo fármaco.

El documento también contiene un práctico algoritmo de manejo de pacientes adultos con infección por COVID-19. El cual está estadificado según si la radiografía de tórax es normal o con neumonía y si requiere manejo ambulatorio u hospitalización según nivel de gravedad.

Esperamos que estas recomendaciones elaboradas en conjunto por tres sociedades cientificas nacionales -SOCHINIF, SOCHIMI y SERChile-sean un aporte al país y sobre todo a los médicos y profesionales de la salud que se encuentran a cargo del diagnóstico y manejo de los pacientes con COVID-19.

Dr. Francisco Arancibia Hernández.

Coordinador Comisión de Infecciones y

Expresidente de la Sociedad Chilena de Enfermedades Respiratorias Jefe Servicio de Medicina Respiratoria del Instituto Nacional del Tórax. Vicepresidente de la Asociación Latinoamericana de Tórax -ALAT. Email:fearancibia@gmail.com

\section{Bibliografía}

1.- DONG E, DU H, GARDNER L. An interactive web-based dashboard to track COVID-19 in real time. Lancet Infect Dis. 2020; 20: 533-4. doi: 10.1016/S14733099(20)30120-1.

2.- DEIS, MINSAL. Estadísticas de defunciones por causas básicas. Disponible en: https://public.tableau. com/profile/deis4231\#!/vizhome/DefuncionesSemanales1_1_16102396688650/DEF?publish=yes.

3.- MINSAL. Informe semanal de defunciones por covid19 n²6, Disponible en: https://www.minsal.cl/wp-content/ uploads/2020/12/Informe-26-Estad\%C3\%ADsticas-deDefunci\%C3\%B3n-Covid19.pdf.

4.- ARROYO C, CORTÉS T, ENGEL E, PARDO W D, SIMONETTI P. Espacio Publico. Informe sobre la evolución de la epidemia de COVID-19 en Chile. Disponible en: www.espaciopublico.cl/wp-content/ uploads/2020/07/CoVid_Chile_23072020_vf.pdf.

5.- GAO J, TIAN Z, YANG X. Breakthrough: Chloroquine phosphate has shown apparent efficacy in treatment of COVID-19 associated pneumonia in clinical studies. BioScience Trends 2020; 14 (1): 72-3.

6.- GAUTRET P, LAGIER JC, PAROLA P, HOANG VT, MEDDEB L, MAILHE M, et al. Hydroxichloroquine and azithromycin as a treatment of COVID-19: results of open-label non-randomized clinical trial. Int J Antimicrob Agents. 2020;56:105949.

7.- US FOOD \& DRUGS ADMINISTRATION (FDA). Letter of Authorization-chloroquine phosphate and hydroxychloroquine sulfate. Disponible en: www.fda. gov/media/136534/download. 
8.- WILSON KC, CHOTIRMALL SH, BAI C, RELLO J. COVID19: Interim Guidance on Management Pending Empirical Evidence. Last updated April 3, 2020. Disponible en: www.thoracic.org.

9.- Retracted coronavirus (COVID-19) papers. Disponible en: https://retractionwatch.com/retracted-coronaviruscovid-19-papers/

10.- OYARZÚN M. Regulaciones en la investigación biomédica en Chile en tiempos de la pandemia por
COVID-19. Rev Chil Enferm Respir 2020; 36 (4): 2435 (editorial).

11.- SOCIEDAD CHILENA DE INFECTOLOGÍA, SOCIEDAD CHILENA DE MEDICINA INTENSIVA Y SOCIEDAD CHILENA DE ENFERMEDADES RESPIRATORIAS. Recomendaciones para el estudio y manejo farmacológico en pacientes adultos con sospecha de infección por SARS-CoV-2 (COVID-19). Rev Chil Enferm Respir 2021; 37 (1): 31-54. 